\title{
Simulation Model of the Scanner Servocontrols for the Orientation of a Fighter Aircraft by Using the Bond Graph Technique
}

\author{
M.L. Martinez, G. Romero, J. Maroto, Felez, J. \\ ETSI Engineering, Universidad Politécnica de Madrid \\ Madrid, Spain \\ e-mail: \{luisa.mtzmuneta, gregorio.romero, joaquin.maroto, jesus.felez\}@upm.es
}

\begin{abstract}
Nowadays the use of complex models has increased the requirements of the simulation tools and techniques for working and analyzing with different domains at the same time. This work focuses on the study of the Scanner servocontrols of a fighter aircraft. The model has the feature of integrating various physical domains into the same system (electrical, mechanical and system control environments) with different dimensions in each domain. The technique used is the Bond Graph (BG), which is typically used in fields where different physical domains coexist, just as in the case we are dealing with, where such domains are fundamental. At the beginning of the study, the different coordinate references are introduced and the physical subsystems are also described. After this the different subsystems are presented in the form of BG submodels one by one and the global Multibond Graph (MBG) model is displayed. Lastly, an example of simulation is shown and some important conclusions are reached.
\end{abstract}

Keywords-Scanner servocontrols, fighter aircraft, Bond Graph, Zero Order Causal paths.

\section{INTRODUCTION}

In this paper we will describe a multidimensional complex Multibond Graph (MBG) which is made up of a fighter aircraft with a radar system. The system is made up of an aircraft rigid body, which displaces and rotates in space, plus a Scanner composed of a gimbal box and an antenna. Motors controlled by servosystems carry out the azimuth and elevation movements of the Scanner. This multibody model has been used to design the servocontrols of the Scanner of a radar which is part of a real fighter aircraft about to go into the production stage. Once the servos have been designed, the model goes on to the parameters tuning process to obtain optimum dynamic responses. The complexity of the application, the assembly of various physical submodels and the existence of various interconnected physical domains has been decisive in choosing Bond Graph (BG) as the simulation tool [1]. The guidelines to obtain the DAE system will be given as well as the results of the simulation.

The developed model includes systems of rigid bodies or Multibody Systems (MBS). The simulation model will lay out the existence of Zero-Order Causal Paths (ZCPs) in a multibond environment. If the possibility is high of algebraic loops appearing in unidimensional systems, the probability increases in MBG systems.

\section{PHYSICAL MODEL OF THE SCANNER}

The model to simulate consists of a free rigid body in space ( 6 degrees of freedom) representing the fighter aircraft. The Scanner is installed in the front of the aircraft and the Scanner is part of it. The gimbal box and the antenna are the moving parts of the Scanner, whose azimuth and elevation movements are controlled by means of servosystems.

Once the MBG model is obtained from the system we will proceed to carry out a simulation of a flight pursuit. The radar system must find a target and the aircraft immediately goes about its maneuver to aim and follow it. The target in the simulation is a point that can move in space with any type of flight track (for example, another aircraft) or it can be in a fixed position (for example, a land object).

\section{A. Coordinate systems}

The inertial reference (global) is fixed on land in the aircraft operation area. Its vectors are defined in a way that the $\mathrm{X}$-axis points towards the North and the $\mathrm{Y}$-axis towards the East. The $\mathrm{Z}$-axis is orthonormal and points towards the center of the Earth.

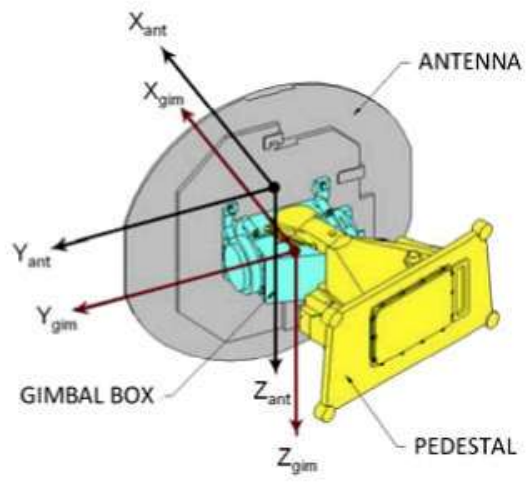

Figure 1. Scanner model

The aircraft reference corresponds to the aircraft's center of gravity, whose origin and orientation is fixed and it can be obtained from a translation of the previous inertial reference. The aircraft orientation reference can be obtained by assigning the rotations $\psi, \theta$ and $\phi$ (heading, elevation and bank) of the aircraft. The $\mathrm{X}$-axis of this base is positioned towards the aircraft's longitudinal direction, the $Y$ - axis is transversal to the aircraft, and the $\mathrm{Z}$-axis is always facing 
downward perpendicular to the plane described by the airframe and wings. The scanner reference (and pedestal) has the same orientation as the aircraft reference but is translated to the crossing of the azimuth and elevation axes of the scanner.

The gimbal box reference is obtained by applying an azimuth rotation $(\alpha)$ to the scanner reference and the antenna reference is obtained by applying an elevation rotation $(\varepsilon)$ to the previous reference system.

\section{B. Description of the subsystems of the simulation}

Any type of maneuver can be carried out on the body of the aircraft. The scanner is basically made up of a pedestal attached to the aircraft bulkhead, a gimbal box which executes an azimuth movement, and the antenna which is hauled by the gimbal box and it can simultaneously execute an elevation movement (fig. 1). Both the azimuth and elevation movements are controlled by the servocontrols.

The pedestal contains the hardware electronics and is used as a support for the moving parts. The gimbal box contains the motors, resolvers, waveguides and the signal and power wiring. It is used as a support for the antenna.

\section{1) Aircraf}

The aircraft angular motion is exerted over a rotational inertia through three Proportional-Derivative controls (type PD). The necessary forces to make the aircraft turn are executed by means of torque feeding over each of the directions defined by the Cardan angles. The translational movement control will also be of PD type and is carried out over the translational inertia of the aircraft. The maneuvers endowed with a high number of g's (gravity acceleration) as well as the aerodynamic forces are performed over the translational inertia which is represented in the inertial reference.

\section{2) Gimbalbox}

It has the capacity of moving in azimuth by means of electric motors and in its movement it hauls the antenna and the antenna arms. At the same time it can rotate in elevation with regard to the gimbal box.

The azimuth motion is executed along the Z-axis of the scanner reference. Notice that the gimbal box's center of gravity does not coincide with the origin of its system reference.

\section{3) Antenna}

Its center of gravity does not coincide with the origin of its system reference either and two arms fix the antenna to the gimbal box. It carries out its elevation motion along the $\mathrm{Y}$-axis of the gimbal box reference.

\section{4) Azimuth Servosystem}

The azimuth servo can be divided into the velocity control, position control and azimuth plant, and the azimuth plant can also be divided into the electromechanical actuator, gearing and load (gimbal box plus antenna).

In this subsystem all the operations are carried out in the $Z$-axis of the scanner reference (and the gimbal box reference).

\section{5) Elevation Servosystem}

The blocks are exactly the same as those of the azimuth, and the parameters of the different elements that are included in the elevation servocontrol are what make them differ.

All the operations in this subsystem are effectuated along the $\mathrm{Y}$-axis of the gimbal box reference (note that the gimbal box reference has been obtained providing the scanner reference with azimuth rotation).

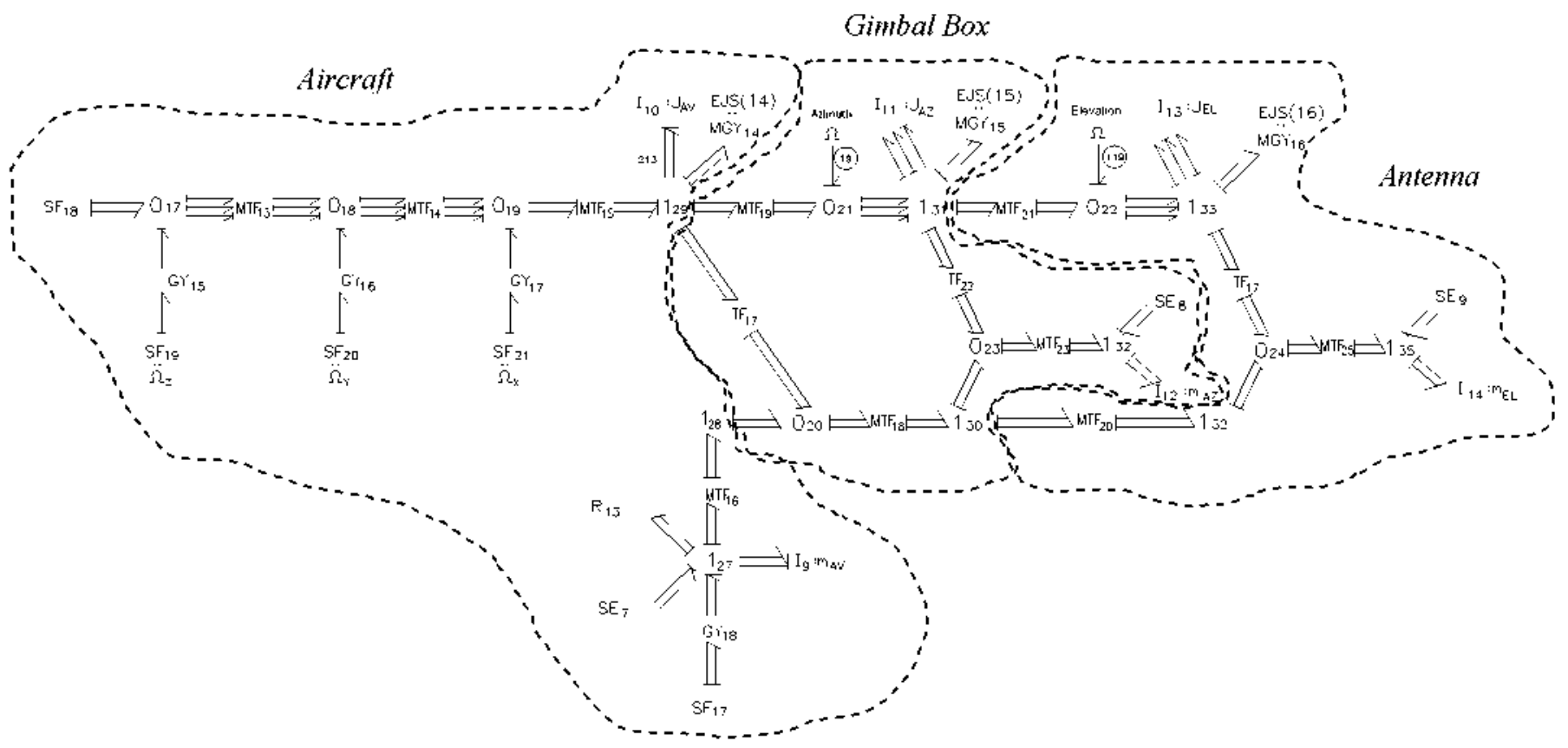

Figure 2. Bond graph nodel of the aircraft and scanner 


\section{Simulation Bond Graph model}

The BG model (fig. 2) is made up of five blocks and each one of them represents a different subsystem (fig. 3).

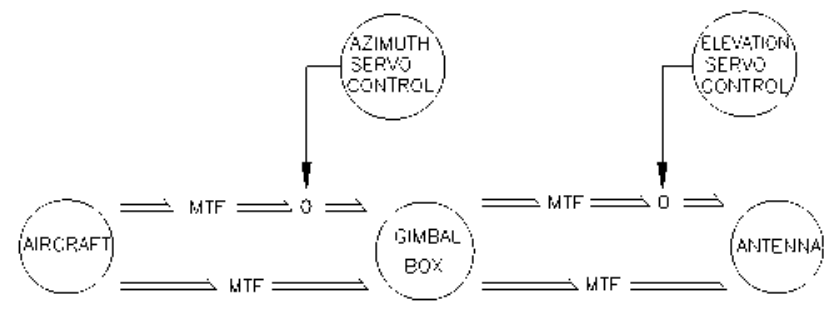

Figure 3. The word bond graph

The coordinate transformations are indicated by the MTF elements. The torques to move the gimbal box and the antenna are input through the 0 -junctions (fig. 3 and junctions $O_{2 f}$ and $O_{22}$ in fig. 2) which represent the angular velocity differences between the aircraft and the gimbal box, and the gimbal box and antenna respectively.

\section{SUBMODELS BOND GRAPH OF THE SYSTEM}

\section{A. Aircraft body}

The subgraph that corresponds to the solid aircraft can be seen in the dashed lines in figure 2 .

It corresponds to the bond graph of a rigid body in space. It has the characteristic that the aircraft must be orientated in space. This is expressed through the Cardan angles between the aircraft reference and the aircraft orientation reference.

Apart from these two references (aircraft position and orientation reference), two auxiliary references are used to determine the intermediate movements of the reference transformations.

The mechanism to move the aircraft in angular position consists of an input torque with a PD control. The torque that is needed to make the aircraft turn is input in the direction of each of the Cardan angles.

The GY elements at the beginning of the bond graph model $\left(G Y_{15,} G Y_{16}\right.$ and $\left.G Y_{17}\right)$ convert the flow sources into torque through amplification ratios. Logically, the higher the GY ratios the faster they will execute the maneuvers, but at the expense of an overshoot which will have to be corrected if the target is to be reached.

The mechanism used to move the aircraft in a translational way also consists of an actuator with a PD control. The necessary effort to make the aircraft move is exerted in each of the directions independently. Thus, the effort input to go forward in each direction is carried out according to the subgraph formed by $S F_{17}, G Y_{18}$ and the 1junction $I_{27}$. Seen from inertia $I_{g}$ these forces represent the acceleration due to the aircraft maneuvers.

The effort source $S E$, represents the aircraft weight and the external disturbances. These values are executed in the inertial reference.

The resistance $R_{13}$ represents the aerodynamic resistances acting on the aircraft. They are considered proportional to the linear velocity. These forces are also expressed in the aircraft inertial reference

\section{B. Gimbal box body}

The subgraph corresponding to the gimbal box is represented with dashed lines in figure 2 .

The linear velocities of the intersection point of the rotation axes of the gimbal box are obtained from the aircraft linear velocities and its angular velocities. These velocities are still expressed in the aircraft orientation reference.

Bond ' 19 ', which is an input port of the 0 -junction $\theta_{2 I}$ in the $Z$ direction of the scanner reference, collects the torque that comes from the azimuth servomechanism. The rotation of the gimbal box causes a change in coordinates from the scanner to the gimbal box reference. The elements $M T F_{18}$ and $M T F_{19}$ carry out this transformation. The transformer $M T F_{23}$ represents the transformation of the gimbal box reference fixed to the gimbal box into the inertial reference.

\section{Antennabodv}

The subgraph corresponding to the antenna body is represented in dashed lines in figure 2 .

Bond ' 119 ', which is the input port of the 0-junction $\theta_{22}$ in the $\mathrm{Y}$ direction of the gimbal box reference, collects the torque that comes from the elevation servomechanism. The antenna rotation produces a change in coordinates from the gimbal box to the antenna reference. The elements $M T F_{20}$ and $M T F_{21}$ carry out this transformation. Finally, the coordinate transformation from the antenna reference fixed to the antenna body up to the inertial reference is carried out by the transformer $M T F_{25}$.

\section{AZIMUTH SERVOSYSTEM}

\section{A. Velocitu control}

The velocity control begins to function when the scanning rate is estimated to be above the maximum velocity permitted at each point of angular scanning. The velocity control (fig. 4) is a proportional control with feed forwards for the velocity demand and the disturbances produced by the aircraft maneuvers.

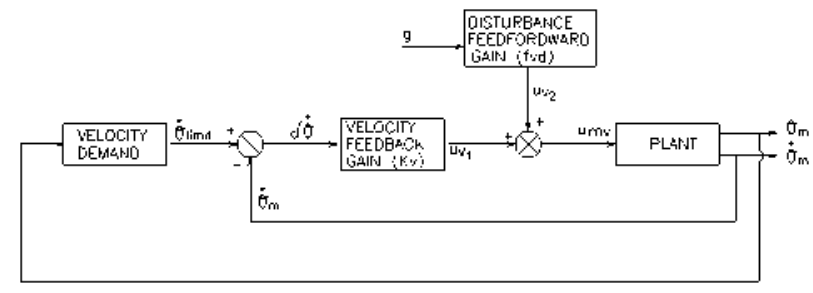

Figure 4. Velocity control block diagram

The notation used is:

$\dot{\theta}$ linit: maximum velocity demand

$\theta \mathrm{n}:$ position measurement

$\dot{\theta}_{\mathrm{m}}$ : velocity measurement

$\delta \dot{\theta}:$ velocity error

$u_{v 1}:$ proportional control tension

$u_{v}$ : disturbances feedforward control tension

$u_{m v}$ : velocity loop control tension

$g$ : disturbance estimate 
The subgraph that corresponds to the azimuth velocity control is represented in figure 5 .

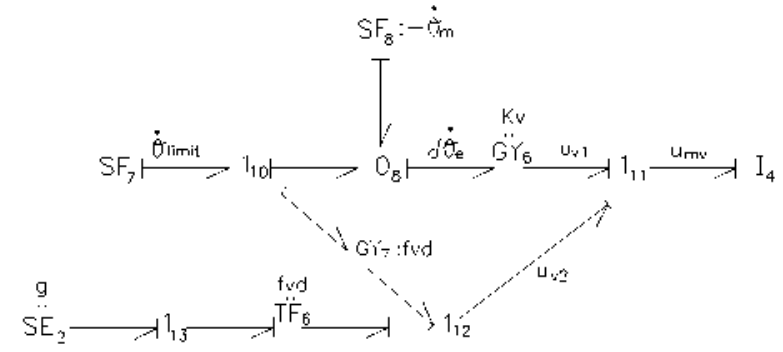

Figure 5. Velocity control subgraph

\section{B. Position control}

It is the normal way of running. The position loop contains a proportional control in position and velocity, a disturbance feed forward and an integral control for the position error; therefore, it is a Proportional-IntegralDerivative (PID) control. The following figure represents its block diagram.

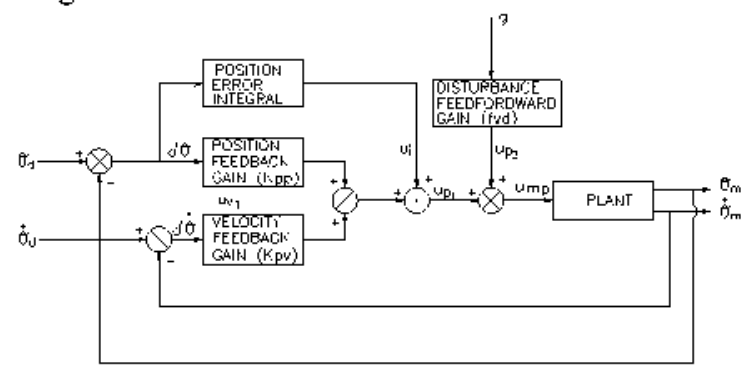

Figure 6. Position control block diagram

The notation used is:

$u_{p l}:$ proportional control tension

$u_{p 2}$ : disturbances feedforward control tension

$u_{m p}:$ position loop control tension

$\vartheta_{d}, \dot{\theta} d:$ position and velocity demand

$\vartheta_{\mathrm{m}}, \dot{\theta} \mathrm{m}$ : position and velocity measurement.

$\delta \theta, \delta \dot{\theta}:$ position and velocity error

$\mathrm{u}_{\mathbf{i}}:$ integral control tension

The subgraph corresponding to the position control is the one that appears in figure 7.

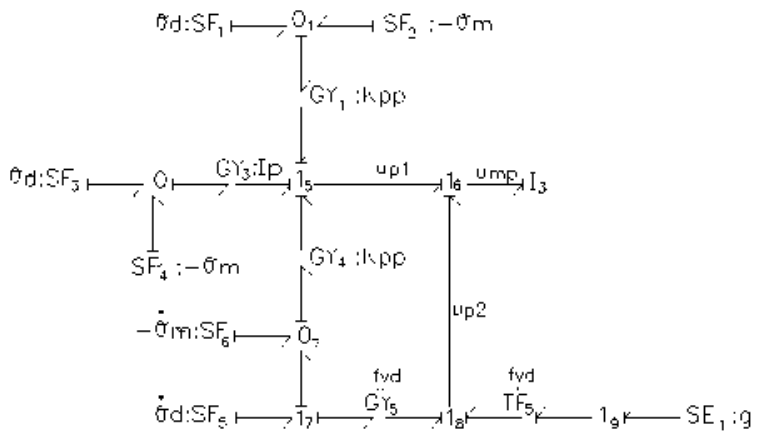

Figure 7. Position control subgraph

\section{Azimuth plant}

This subsystem which also includes the amplifier, motor and gearbox, gearing and load subsystems is represented in the corresponding subgraph in the next figure. Source $S E_{3}$ corresponds either to $u_{m p}$ or $u_{m v}$. depending on what servosystem is used.

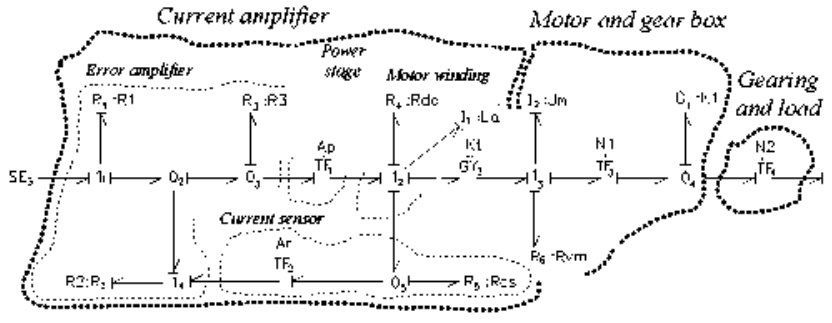

Figure 8. Azimuth plant subgraph

\section{1) Current amplifier}

It is the error amplifier, power stage, and motor winding plus current sensor all together. The following figure represents its block diagram.

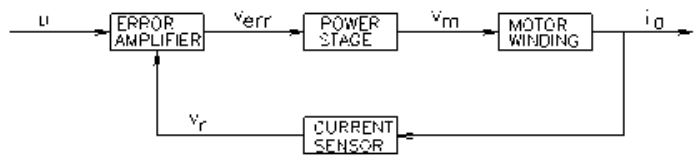

Figure 9. Current amplifier block diagram

The outline of the error amplifier block is the one represented in figure 10 .

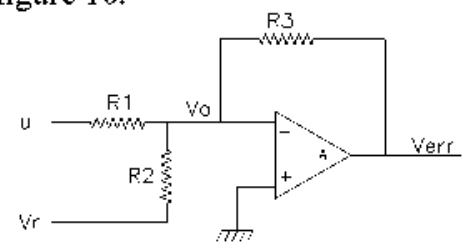

Figure 10. Error anplifier electric scheme

The power stage can be considered in the model simply as an amplification coefficient that is the ratio $A_{p}$ in element $T F_{1}$. The voltage at the output of the power stage is what feeds the motor $\left(v_{m}\right)$.

The motor winding block is represented in the following figure:

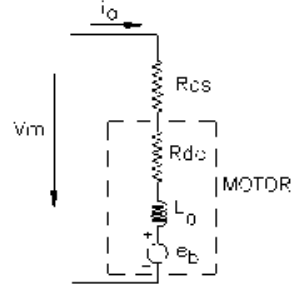

Figure 11. Motor winding electric scheme

, where $R_{d c}$ is the resistance of the motor winding, $L_{a}$ is the winding inductance, and $e_{b}$ is the back e.m.f.

Finally, the current sensor through the resistance $R_{c s}$ uses a differential amplifier to amplify the signal. 


\section{2) Motor and gear box}

The output torque of the motor is proportional to the current through its coils $\left(i_{s}\right)$ and is represented in figure 8 in the output port of element $G Y_{2}$.

This element $G Y_{2}$ is also used as the passing-through port of the electric domain to the mechanic. The torque moves the actuator rotor that has inertia $J_{m}$

The motion of the inertia is constrained by the resistance which offers the viscose friction $R_{v m}$, represented by the linear resistance $R_{6}$.

The load in the rotor has been simulated as a torsional spring (element $C_{l}$ ). Its stiffness corresponds to the gearbox that is integrated in the output shaft of the motor.

\section{3) Gearing and load}

The gearing transmits the torque from the exit of the gearbox to the load. This gearing has a transmission ratio $N_{2}$ and is represented by element $T F_{+}$in figure 8 .

The output torque of this gearing will move the load. In the bond graph model this torque (effort in bond ' 19 ', fig. 8) acts, just as in the entire azimuth servo, in the Z-direction of the scanner reference. From this bond on, the model will be multibond and will form part of the mechanic domain. The subsystem it connects to is the gimbal box body.

\section{Elevation Servosystem}

This subsystem is structurally similar to the previous subsystem, for which reason the explanation and graph are omitted. The bond that provides the torque to move the antenna in elevation is the number ' $119^{\prime}$ ' (fig. 8) and it connects with the antenna body. No further explanation is necessary.

\section{DERIVING THE MATHEMATICAL MODEL}

Once the BG model is causally augmented, the existence of ZCPs is detected. The way of resolving the system will be by searching for the break variables that open the topological loops. This gives way to a DAE equation system. In spite of being relatively old [2], this method has not been further utilized until mathematical methods capable of resolving DAE systems [3] came up. In [4] and [5] the method is introduced, the ZCPs are systematized and classified. In [6] the method is extended to MBG models and the new classes 5 of ZCPs are introduced.

The algorithms for tearing the topological loops in MBG with a minimum number of break variables are described in [7], where a great number of examples are included. As a result of the application of these algorithms, a minimum number of additional restriction equations are obtained. These relate the break variables (semi-state variables) to themselves through algebraic assignments. These equations, which are used to break all the topological loops, are responsible for the system becoming a DAE.

The stages to obtain the mathematical model are the causality augmentation of the BG model, the detection of existing ZCPS in the model and the topological loops opening, in which the break variables are generated that give way to algebraic equations, and the application of the mathematical algorithm to the DAE system.

\section{EXAMPLE OF SIMULATION}

An application whose objective is for the fighter aircraft to detect a moving target (for example, a missile) is proposed. At the beginning of the simulation, the target moves around in any type of trajectory (fig. 12.a) and once it is in the visual field of the radar (fig. 12.b), the aircraft must shift in a way in which its direction points at the target. Simultaneously the aircraft must move in the space searching for the objective up to a certain distance or shooting zone (fig. 12.c), and once it is there, it shoots and begins to move away (fig. 12.d). The aircraft will always try to keep the target inside the radar field.

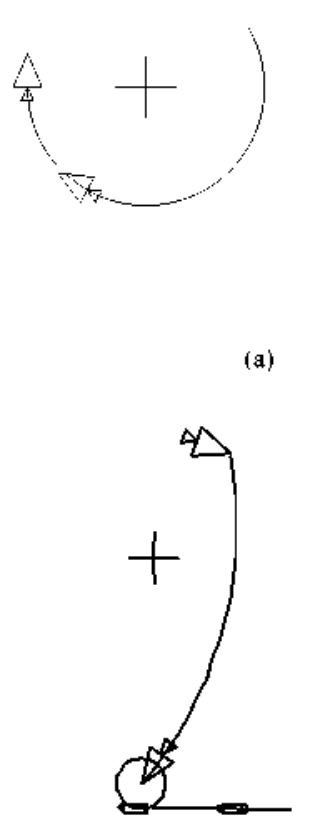

(c)

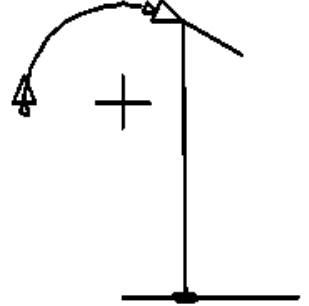

(b)

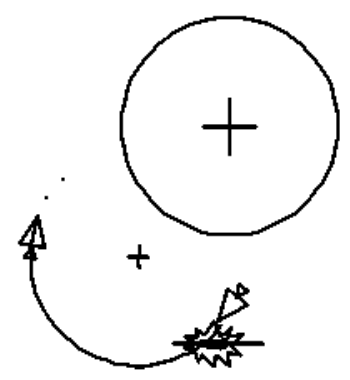

(d)
Figure 12, Simulation steps

The radar might not detect any objective in its action field or the target might get away due to a sharp and rapid maneuver on the target's part. In these cases the aircraft will stay in a parallel "orbit" to land (X, Y-axis of the inertial base). It will then begin to make circles of wide radius. Now the radar will carry out sinusoidal scanning in azimuth and elevation searching for the same objective or a new one. Once the target is detected, the Scanner will begin to point towards it. This will avoid it from losing the target. It is obvious that the target will not be detected if it is above or below the aircraft and inside the dead angle of the radar.

\section{A. Simulation results}

All the aircraft as well as the scanner displacements and rotations that are executed in figure 13 have been obtained as a function of simulation time. Thus, the pursuit can be represented as an animated graph.

Figure 13 shows the curve of the aircraft displacements in both axes. This figure summarizes the four phases previously described. 


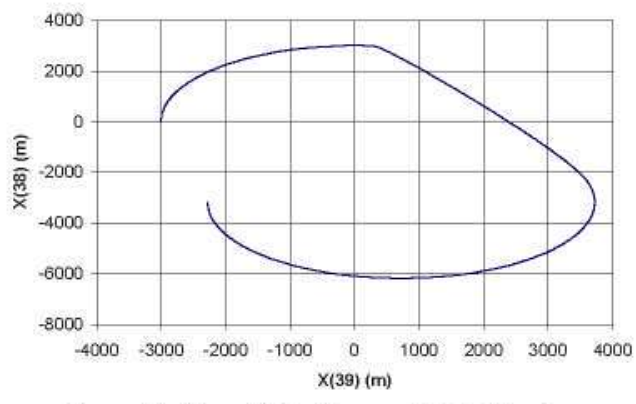

Figure 13. Aircraft displacement in $\mathrm{X}-\mathrm{Y}$ axis

Finally, figure 14 shows the scannings in azimuth carried out by the Scanner. In the non-fluctuating stage the target remains inside the radar field.

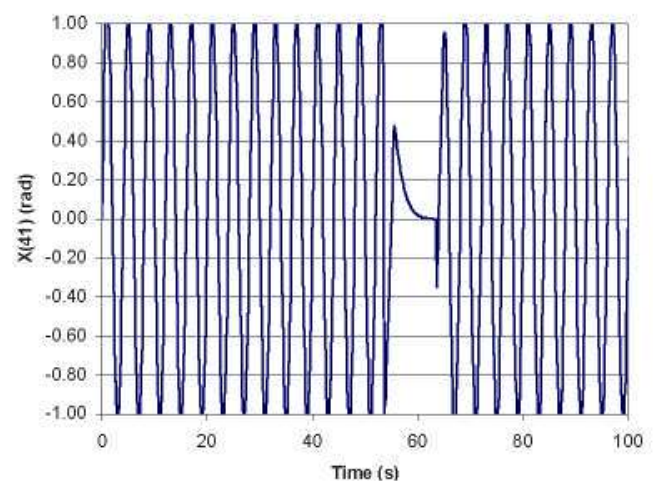

Figure 14. Azimuth sweep of the Scanner

\section{CONCLUSIONS}

A BG model of a fighter aircraft with a radar system has been developed. The final model turns out to be an MBS system with interconnected bodies. The simulation example consists of detecting a target in space through the Scanner and going after it.

This paper has various objectives. The first of which is to highlight the advantage that the $\mathrm{BG}$ technique has for simulating in a simple way systems that are part of different physical domains. The second is to show how easily physical submodels which have relative movements among themselves and whose output signals are used as inputs for others are easily assembled. [8] standardizes in a simple manner the modeling of bodies through MBG, which is an important study carried out by the author.

\section{REFERENCES}

[1] D.C. Karnopp, D.L. Margolis, R.C. Rosenberg. System Dynamics: a Unified Approach. 2nd Edition, Wiley Interscience, New York, 1990.

[2] G. Kron. Diakoptics - The piecewise solution of large-scale systems, MacDonald \& Co., London. 1962.

[3] C.W.Gear. The simultaneous numerical solution of differentialalgebraic equations. IEEE Trans. Circuit Theory, Vol.18, pp.89-95. 1971.

[4] J. Van Dijk. On the role of bond graph causality in modeling mechatronic systems. Ph.D.Thesis. Twente University, Enschede, The Netherlands. 1994.
[5] J. Van Dijk and P. Breedveld. Simulation of System Models Containing Zero-order Causal Paths- I. Classification of Zero-order Causal Paths. Journal of the Franklin Institute. Vol. 328, No. 5/6, pp. 959-979. 1991.

[6] R. Cacho, J. Félez, C. Vera. Deriving simulation models from bond graphs with any combination of topological loop classes. International Conference on Bond Graph Modeling and Simulation, Phoenix. SCS, Vol. 29, No. 1. 1997.

[7] G. Romero, J. Félez, C. Vera. Optimised Procedures for Obtaining the Symbolic Equations of a Dynamic System by Using the Bond Graph Technique. International Conference on Bond Graph Modeling and Simulation, Phoenix. SCS, Vol. 37, No. 1. 2005.

[8] A.M. Bos. Modeling Multibody Systems in terms of Multibond Graphs. Ph.D.Thesis, Twente University, Enschede, The Netherlands. 1986. 